American Journal of Agricultural and Biological Sciences 4 (3): 187-191, 2009

ISSN 1557-4989

(C) 2009 Science Publications

\title{
Quantitative Performance of Spectral Indices in Large Scale Plant Health Analysis
}

\author{
Helmi Zulhaidi Mohd Shafri and Mohanad Saad Ezzat \\ Department of Civil Engineering, Faculty of Engineering, \\ University Putra Malaysia, 43400 Serdang, Selangor, Malaysia
}

\begin{abstract}
Problem statement: Oil palm trees are planted in large scale areas. The detection and mapping of diseases are considered as important for oil palm industry and need a timely detection to control the disease spread. Approach: Vegetation analysis of airborne hyperspectral imagery could be an ideal method to deal with this problem since this data could be acquired on user demand. Airborne hyperspectral dataset was preprocessed in order to prepare it for the vegetation analysis processing for the purpose of detection and mapping Ganoderma disease in oil palm trees. Many vegetation indices were tested and analyzed to classify oil palm trees into healthy and unhealthy trees, in both individual analysis of vegetation indices and forest health composites that are available in ENVI software. Accuracy assessment was calculated by using ground truth data. Results: The results showed that all vegetation indices tested in this study provide a good accuracy which ranges from 68.57-82.86 and 60$80 \%$ for both vegetation indices and forest health composites respectively. With regard to the vegetation indices the highest accuracy was achieved by using Red Edge Normalized Difference Vegetation Index (NDVI 705) with 82.86\% of overall accuracy and as for the forest health composites the highest accuracy was achieved by using the composite that include Vogelmann Red Edge Index 1 (VOG1) with $80 \%$ of overall accuracy. Conclusion/Recommendations: Vegetation indices based on the red edge provide better results than other indices based on other techniques.
\end{abstract}

Key words: Airborne sensor data, oil palm, Ganoderma disease, red edge

\section{INTRODUCTION}

Vegetation is a fundamental element of the earth's surface and has a major influence on the exchange of energy between the atmosphere and the earth's surface $^{[1]}$. Over the years the quantity and variability of vegetation biomass have been estimated indirectly by use of vegetation indices derived from remotely sensed data. In most vegetation studies, vegetation indices calculated using the red and near infrared band of broad band. Broad band indices are useful for vegetation status $^{[2]}$. Although broad band indices have many advantages for vegetation studies, they are affected by factors such as soil brightness, soil color, the atmosphere, sensor calibration, bi-directional reflectance and differences in spectral resolution of sensors. In order to account for these differences, specific indices have been developed. Among them is the Normalized Differences Vegetation Index which has been widely applied ${ }^{[3]}$. Generally, these indices have been used to assess green plants based on their spectral reflectance values ${ }^{[2]}$. Application of remote sensing has been one of the challenges to many scientists especially in quantifying vegetation biomass at landscape level. Accurate quantitative estimation of vegetation biochemical and biophysical characteristics is necessary for a large variety agricultural, ecological and meterological applications ${ }^{[4,5]}$. Likewise, the mapping and monitoring of vegetation biochemical and biophysical variables is important for the spatially distributed modeling of vegetation productivity, evapotranspiration and the surface energy balance ${ }^{\left[[]^{6}\right.}$. Remote sensing may be used to detect vegetation changes. The reflectance of healthy vegetation in the visible light is characterized by absorption features caused by plant pigments such as chlorophyll and carotenoids, while the Near Infrared Regions (NIR) and Shortwave Infrared Regions (SWIR) are characterized by four major water absorption features.

Quantifying vegetation biomass using vegetation indices based on broad band sensor data is still a problem. Most broad band sensors have data with coarse spectral resolution thereby averaging reflectance over a wide wavelength. Therefore, a major drawback

Corresponding Author: Helmi Zulhaidi Mohd Shafri, Department of Civil Engineering, Faculty of Engineering, University Putra Malaysia, 43400 Serdang, Selangor, Malaysia 
of traditional remote sensing products in the loss of crucial information available in specific narrow bands ${ }^{[7,8]}$. In this regard, the advent of hyperspectral remote sensing has offered possibilities to overcome this limitation. The tools for vegetation remote sensing have developed considerably in the past decades ${ }^{[4]}$. Optical remote sensing has expanded from the use of multi-spectral sensors to that of imaging spectrometers. Imaging spectrometry or hyperspectral remote sensing, with sensors that typically have hundreds of narrow, contiguous spectral bands between 400 and $2500 \mathrm{~nm}$, has the potential to measure specific vegetation variables that are difficult to measure using conventional multi-spectral sensors. Since most of the researchers had been in the area of foliar biochemical estimation ${ }^{[9]}$. After the introduction of spectroscopy in vegetation science researchers have focused not only on foliar chemical estimation but also on plant biophysical characteristics. Since spectrometers have finer spectral resolution and high spatial resolution. Vegetation indices have been used in plant stress detection. Many studies have focused on the use of vegetation indices, Vegetation indices are widely used for the estimation of crop and vegetation variables and stress by using visible and Near Infrared Regions (NIR) of the electromagnetic spectrum. Healthy plant typically displays very low reflectance and transmittance in visible region and very high reflectance and transmittance in $\mathrm{NIR}^{[10]}$. A vegetation index is defined as a dimensionless, radiometric measure that functions as an indicator of relative abundance and activity of green vegetation, often including Leaf Area Index (LAI), percent green cover, chlorophyll content, green biomass and Absorbed Photosynthetically Active Radiation $(\mathrm{APAR})^{[11]}$. In this study, airborne hyperspectral imagery has been used for Ganoderma disease detection and mapping. Airborne hyperspectral can acquire data in narrow and contiguous spectral bands which makes it possible to discriminate between healthy and diseased plants better compared to multispectral imagery. By using vegetation indices and forest health composites oil palm trees could be classified into healthy and unhealthy trees accurately.

\section{MATERIALS AND METHODS}

The study area is located in Selangor Malaysia. The dataset is a hyperspectral image was taken using the Airborne Imaging Spectroradiometer for Applications (AISA). AISA airborne hyperspectral imaging spectrometer is capable of collecting data within a spectral range of $430-900 \mathrm{~nm}$. This image contains 20 bands with wavelength ranging from 433-
$839 \mathrm{~nm}$. The spatial resolution is 1 meter while the spectral resolution is $6.43 \mathrm{~nm}$. Figure 1 shows the overall flow chart of the methodology and all the steps that had been performed in this study. As for the preprocessing of the dataset CaliGeo software was used to perform Geometric and radiometric corrections to the raw AISA data while ENVI is providing the tools for the data analysis and interpretation. CaliGeo is a separate software package under ENVI software environment designed to process raw AISA sensor data to a format that can be read into any modern data analysis package. The MNF Rotation transforms were applied to determine the inherent dimensionality of image data, to segregate noise in the data and to reduce the computational requirements for subsequent processing. The Cross-Track Illumination Correction also was applied to remove variation in the cross-track illumination of an image. Cross track illumination variations may be due to vignetting effects, instrument scanning, or other non-uniform illumination effects. With regard to vegetation analysis the processing part involved calculation of vegetation indices and forest health composites.

For vegetation indices, it can be divided into three categories which are Broadband Greenness, Narrowband Greenness and Light Use Efficiency vegetation indices. While for forest health composites they consist of three indices from different categories. All the calculations of the vegetation indices and forest health composites were done using 'vegetation analysis' tool in ENVI 4.4 software. They were used in

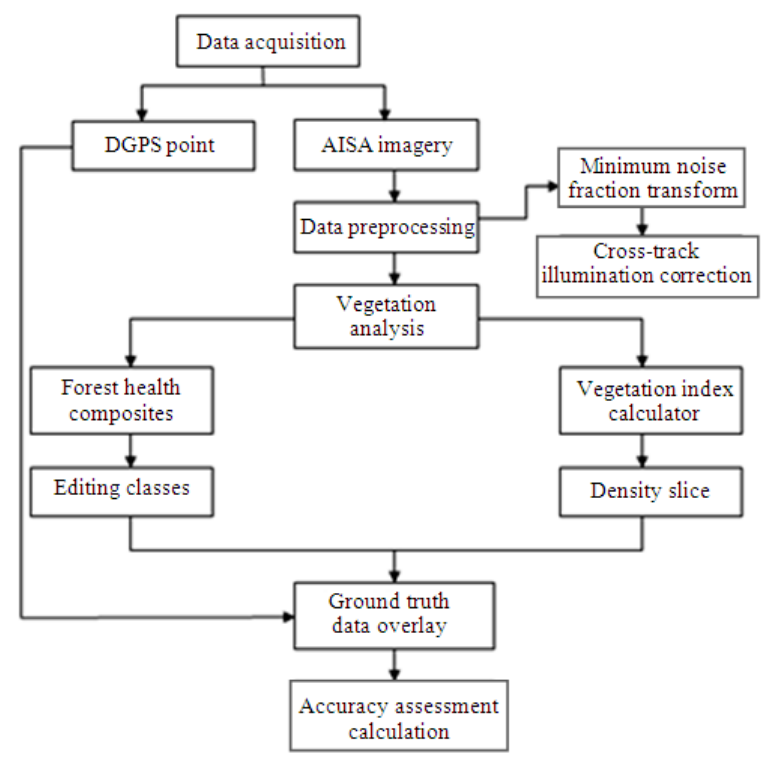

Fig. 1: Methodology flowchart 
further processing to classify oil palm trees in the image into healthy and unhealthy. After that, by overlaying 35 Differential Global Positioning System (DGPS) points which were measured for unhealthy oil palm trees to the classification results, accuracy assessment was calculated for each classification result in order to identify the best vegetation index and forest health composite based on the highest overall accuracy.

\section{RESULTS}

By examining the vegetation indices results, it has been found that the Red Edge Normalized Difference Vegetation Index (NDVI 705) provides the highest accuracy with $82.86 \%$ overall accuracy. Figure 2 shows the NDVI 705 classified map. This index is categorized under the narrowband vegetation indices category. The red edge vegetation index has been used to detect Ganoderma disease in oil palm with much more accurate results than the traditional broadband vegetation indices, whereas the accuracy assessment for the broadband indices were 80 and $71.43 \%$ for both the Simple Ratio (SR) index and the Normalized Difference Vegetation Index (NDVI) respectively. The red edge vegetation index also provides better results than the Light Use Efficiency indices whereas, the accuracy assessments of the Light Use Efficiency indices were $77.14,71.43$ and $68.57 \%$ for the Structure Insensitive Pigment Index (SIPI), Red Green Ratio (RG Ratio) index and Photochemical Reflectance Index (PRI) respectively. Figure 3 shows all the vegetation indices results with their accuracy assessments.

By examining the forest health composite results, it has been found that the best forest health composite was the composite that consists of Vogelmann Red Edge Index 1 (VOG1), Anthocyanin Reflectance Index 1 (ARI1) and Structure Insensitive Pigment Index (SIPI) with overall accuracy of $80 \%$. Figure 4 shows the best Forest Health Composite classified map.

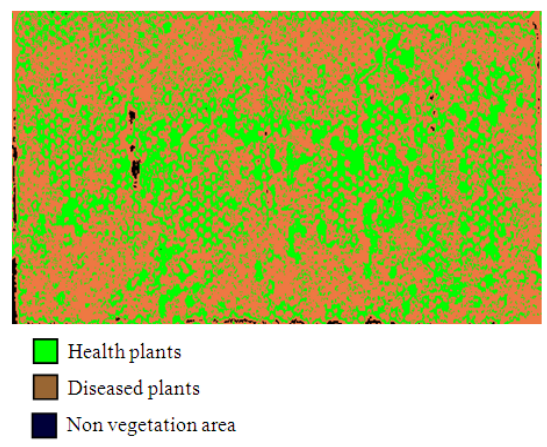

Fig. 2: NDVI 705 classified map
The Vogelmann Red Edge Index 1 (VOG1) is categorized under the narrowband vegetation indices category. Forest health composites that consist of VOG1 narrowband vegetation index have been used to detect Ganoderma disease in oil palm with much more accurate results than the Forest health composites that consist of broadband vegetation indices, whereas, the accuracy assessments of the broadband indices were 71.43, 62.86 and $60 \%$ by using both the Simple Ratio (SR) index and the Normalized Difference Vegetation Index (NDVI) with Structure Insensitive Pigment Index (SIPI), Photochemical Reflectance Index (PRI) and Red Green Ratio (RG Ratio) respectively. Forest health composites that consist of VOG1 also provide better results than the Forest health composites that consist of Red Edge Normalized Difference Vegetation Index (NDVI 705) narrowband vegetation index whereas, the accuracy assessments of the NDVI 705 index were 71.43, 65.57 and $60 \%$ with the using of Red Green Ratio (RG Ratio) index, Photochemical Reflectance Index (PRI) and Structure Insensitive Pigment Index (SIPI) respectively.

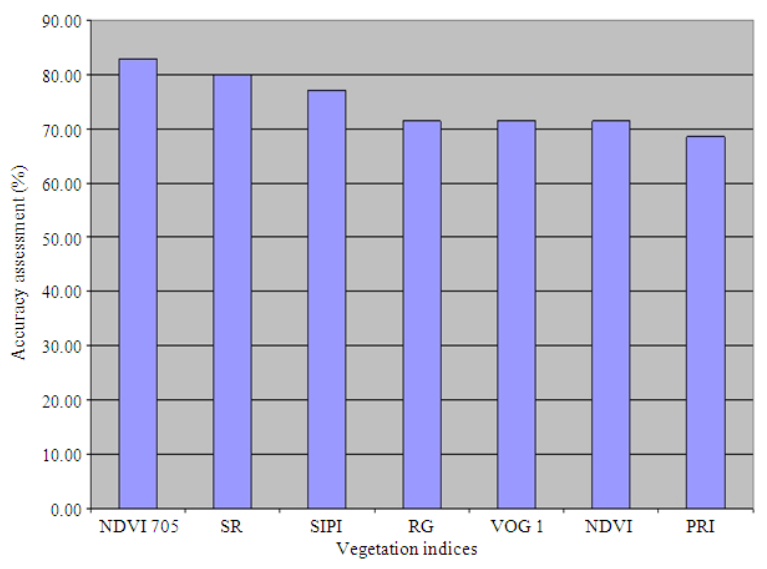

Fig. 3: Vegetation indices results with their accuracy assessments

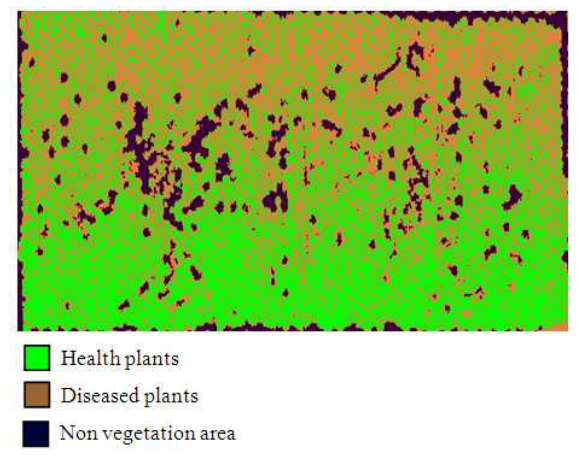

Fig. 4: Best forest health composite classified map 


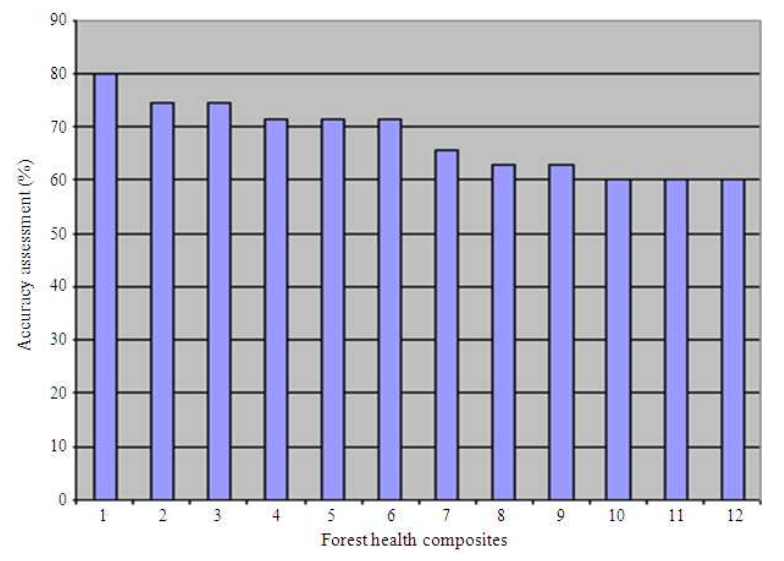

Fig. 5: Forest health composite results with their accuracy assessments

On the other hand by examining the forest health composites, it has been found that the composites that consist of the broadband index, Leaf Pigments index and Light Use Efficiency index provide no difference in the results even with the change of the broadband index and maintain both of the Leaf Pigments index and Light Use Efficiency index. In contrast there is a significant change in the results by changing the Light Use Efficiency index and maintain the broadband index. The accuracy assessments were 71.43, 62.86 and $60 \%$ by using the Light Use Efficiency indices Structure Insensitive Pigment Index (SIPI), Photochemical Reflectance Index (PRI) and Red Green Ratio (RG Ratio) respectively with both of Normalized Difference Vegetation Index (NDVI) and Simple Ratio (SR) broadband indices. Figure 5 shows all the forest health composite results with their accuracy assessments.

\section{DISCUSSION}

The results show that both vegetation indices and forest health composites highest results were obtained by using vegetation indices under the narrowband category. Since these indices are categorized under the narrowband vegetation indices they are intended for use with very high spectral resolution reflectance data, such as from hyperspectral sensors. These indices capitalize on the sensitivity of the vegetation red edge to small changes in canopy foliage content, gap fraction and senescence. This wavelength is related for instance to the chlorophyll concentration and the leaf water content which play a main role in determination and classification vegetation conditions. The best result of the forest health composites also consists of the Structure Insensitive Pigment Index (SIPI) which is a reflectance measurement designed to maximize the sensitivity of the index to the ratio of bulk carotenoids to chlorophyll while decreasing sensitivity to variation in canopy structure. Thus, the SIPI index becomes a function of chlorophyll and carotenoid and the effect of the structural components should be eliminated. Moreover, the SIPI index improves the result by minimizing the effects of the surface and leaf internal mesophyll structure that could confound the variability in pigment concentrations.

Obviously forest health composite results proved that the broadband indices could not recognize the small changes in vegetation conditions while the red edge indices have the ability to recognize these small changes. This fact has been proven based on the results of forest health composites that consist of broadband indices which show there is no difference in the results by changing the broadband indices. In contrast there is a significant difference in the results by changing the light use efficiency indices and maintain the broadband indices. Therefore, the advantage of the red edge parameter over broadband spectral indices such as SR and ND is that it is not affected by additive constants such as leaf surface reflectance. Thus, it would be expected to be more reliable when the data includes a wide range of leaf structures.

\section{CONCLUSION}

Ganoderma disease was detected and classified in the oil palm plantation by analyzing vegetation indices available in the ENVI software. The results were analyzed by using both vegetation index calculator and forest health tools respectively for the purpose of Ganoderma disease detection. The accuracy assessment of the classified maps was calculated by overlaying 35 Differential Global Positioning System (DGPS) points which measured for unhealthy oil palm trees to the classification results. The statistical results show that, both vegetation index calculator best result and forest health tools best result involved indices that are based on the red edge calculations which were the Red Edge Normalized Difference Vegetation Index (NDVI 705) and Vogelmann Red Edge Index 1 (VOG1) for both vegetation index calculator and forest health tools respectively. In vegetation applications, the advantage in using narrow bandwidths in the red to near infrared area is the possibility to define smaller changes in the red edge feature. In conclusion vegetation indices based on the red edge provide better results than other indices based on other techniques. 


\section{REFERENCE}

1. Bacour, C., S. Jacquemoud, Y. Tourbir, M. Dechambre and J.P. Frangi, 2002. Design and analysis of numerical experiments to compare four canopy reflectance models. Remote Sens. Environ., 79: 72-83. http://cat.inist.fr/?aModele=afficheN\&cpsidt=1339 8891

2. Maselli, F., 2004. Monitaring forest conditions in a protected Mediterranean coastal area by the analysis of multiyears NDVI data. Remote Sens. Environ., 89: 423-433. http://cat.inist.fr/?aModele $=$ afficheN\&cpsidt $=1552$ 6570

3. Haboudane, D., J.R. Miller, E. Pattey, P.J. Zarco-Tejada and I. Strachan, 2004. Hyperspectral vegetation indices and novel algoryhtms for predicting green LAI of crop canopies: Modeling and validation in the context of precision agriculture. Remote Sens. Environ., 90: 337-352. DOI: 10.1016/j.rse.2003.12.013

4. Asner, G.P., 1998. Biophysical and biochemical sources of variability in canopy reflectance. Remote Sens. Environ., 64: 234-253. http://cat.inist.fr/?aModele $=$ afficheN\&cpsidt $=2299$ 347

5. Houborg, R., H. Soegaard and E. Boegh, 2007. Combining vegetation index and model inversion methods for the extraction of key vegetation biophysical parameters using Terra and Aqua MODIS reflectance data. Remote Sens. Environ., 106: 39-58.

http://dialnet.unirioja.es/servlet/articulo?codigo $=21$ 85555
6. Turner, D.P., W.B. Cohen, R.E. Kennedy, K.S. Fassnacht and J.M. Briggs, 1999. Relationships between leaf area index and landsat TM spectral vegetation indices across three temperate zone sites. Remote Sens. Environ., 70: 52-68.

http://cat.inist.fr/?aModele $=$ afficheN\&cpsidt $=1204$ 303

7. Blackburn, G.A., 1998. Quantifying chlorophyll and caroteniods at leaf and canopy scales: An avalution of some hyperspectral approaches. Remote Sens. Environ., 66: 273-285. http://cat.inist.fr/?aModele $=$ afficheN\&cpsidt $=1657$ 394

8. Thenkabail, P.S., R.B. Smith and E. De pauw, 2000. Hyperspectral vegetation indices and their relationship with agricultural crop characterstcs. Remote Sens. Environ., 71: 158-182. http://cat.inist.fr/?aModele $=$ afficheN\&cpsidt $=1260800$

9. Van der Meer, F.D., 2004. Analysis of spectral obsorption features in hyperspectral imagery. Int. J. Applied Earth Observ. Geoinform., 5: 55-68. DOI: 10.1016/j.jag.2003.09.001

10. Shafri, H.Z.M. and N. Hamdan, 2009. Hyperspectral imagery for mapping disease infection in oil palm plantation using vegetation indices and red edge techniques. Am. J. Applied Sci., 6: 1031-1035. http://www.scipub.org/fulltext/ajas/ajas6610311035.pdf

11. Jensen, J.R., 2000. Remote Sensing of the Environment: An Eartch Resources Perspective. Prentice-Hall, Inc., ISBN: 10: 0134897331, pp: 544. 